\title{
Graphene and related 2D materials for desalination: A review of recent patents
}

\author{
M.W. Aqra and A.A. Ramanathan ${ }^{2 *}$ \\ ${ }^{1}$ National Center for Research and Development, Amman-11941, Jordan \\ Email: maha aqra@yahoo.com \\ ${ }^{2}$ The University of Jordan, Amman-11942, Jordan; \\ Email: amallahmad@gmail.com *corresponding author
}

\begin{abstract}
The growing population and energy demand, coupled with the depleting fresh water resources has resulted in great progress in sea water desalination (SWD) technologies. Nanopores of 2D materials like graphene and its structural analogs are the latest innovations in membrane technology for SWD. The performance of these novel atomically thin nanopores as seen from various experimental and theoretical studies is highly encouraging with reports of water permeability 2-3 orders of magnitude greater than the conventional reverse osmosis (RO). The potential for high efficiency and the low energy requirements of these nanopores for desalination has led to tremendous efforts in fabrication and commercialization. We present here a review of the very recent patents associated with the preparation of these nanopores, the process and the efficiency of SWD.
\end{abstract}

Key words: 2D nanopores; graphene; membrane; patents; desalination.

\section{Introduction}

It is expected that by 2030 more than $15 \%$ of the world population will be forced to turn to seawater to meet their needs for fresh or brackish water. At present around 150 countries rely on desalination and around 80 million $\mathrm{m}^{3}$ of drinking water is being produced daily by more than 17,000 desalination plants, with $50 \%$ of them utilizing sea water as the source (Wali 2014). The desalination process needs to be perfected and made more energy efficient, cost effective, environment friendly and sustainable (Elimelech and Phillip 2011; Pendergast and Hoek 2011; Zhu et al 2013 and Yuan et al 2015). The rapid and innovative advancements in nanotechnology during the past decade and the quick strides taken in nanoscale fabrication has given rise to the discovery of nanopores of atomically thin membranes of graphene and other 2D materials that can filter molecules and selectively transport ions in nano-channels for various applications in energy, sensing, medicine and desalination (Nguyen and Nguyen, 2016; Thanh et al, 2018; Wasfi et al, 2018; Moghadam and Park 2018; Ramanathan et al 2018).

Nano-filteration (NF) and reverse osmosis (RO) that use filters are limited by the low permeability of the filters. Even though today RO is most established method and the efficiency of RO is thrice what it was two decades ago, it is a slow diffusion process. Whereas, nanopores use a sieving method and fast water transport in an efficient manner with high rates of salt rejection is possible (Suk et al 2008; Hilder et al 2009; Suk and Aluru 2010; Tanugi-Cohen and Grossman 2012; Surwade et al 2015; 
Heiranian et al 2015; Wu et al 2015; Li et al 2016). For efficient desalination a membrane must demonstrate a high salt rejection rate along with a high water flux. High permeable membranes can be achieved by engineering the right pore size, chemical functionalities and other surface characteristics (Suk and Aluru 2010; Tanugi-Cohen and Grossman (2012, 2014); Surwade et al 2015; Tanugi-Cohen et al 2016). Nanoporous graphene (NPG) has a separation rate of two-three orders of magnitude higher than diffusive RO as shown by the molecular dynamics (MD) studies (Suk and Aluru 2010; Tanugi-Cohen and Grossman 2012). In addition, the experimental study of (Surwade et al 2015) for single layer graphene shows almost $100 \%$ salt rejection and rapid water fluxes of $10^{6}$ $\mathrm{g} / \mathrm{m}^{2} \mathrm{~s}$ at $40^{\circ} \mathrm{C}$. These results confirm NPG as a desalination device with a very high potential. Further, first principles studies of Molybdenum disulphide $\left(\mathrm{MoS}_{2}\right)$ give $70 \%$ higher water fluxes than NPG and allow for strain tuning of the membrane (Surwade et al 2015; Heiranian et al 2015). Again, simulation studies of boron nitride (BN) show higher efficiency than NPG and superior water flow and salt rejection performance (Suk et al 2008; Hilder et al 2009; Wu et al 2015). The insights obtained by theoretical findings regards the optimum pore size, functionality and material design must be translated into practice for fabrication and manufacture of optimal desalination devices. The recent years have seen several innovative techniques of nanoporous membrane preparation and patents in the various processes, methods and materials for desalination.

Water is a scarce resource and SWD is the most sustainable and attractive option for water management. In this context, the past decade has seen a tremendous increase in research on the use of nanopores of 2D materials like graphene and transition metal dichalcogenides for energy efficient desalination technologies. Now, that this novel technology is ripe for commercial implementation, it is important to bring to the attention of the scientific community the recent patents on this valuable desalination technology. In addition to being energy efficient, nanopores offer water permeability 23 orders of magnitude greater than the conventional reverse osmosis. Since this is a rapidly developing field with exciting new scientific findings, the focus of the present review article is on the recent (since 5 years) patents in this new and exciting field of graphene and related 2D material nanopores. The immense possibilities and success in water desalination using this novel technology is highlighted with some interesting patents and discussions of the various aspects concerning the preparation, process and fabrication of nanoporous optimal desalination devices. The paper is organized under the headings Introduction, Structure of Nanopores and patents therein, Process, performance and effectiveness of nanopores and Challenges and outlook.

\section{Structure of Nanopores and patents therein}

Patents (Choi et al 2012; Sinsabaugh et al 2015; Stoltenburg et al 2017) deal with various ways of making nanoporous membranes of graphene and related 2D materials. In the patent (Choi et al 2012), graphene separation membrane with a plurality of grain boundaries on a polymer support has been prepared for separation of ions in water (desalination) and in gases. The polymer support may include polysulfone, polyethersulfone, polyimide, polyamide, polyetherimide, polyacrylonitrile, polyethylene, polycarbonate, polytetrafluoroethylene, polypropylene, or polyvinylidene fluoride. The separation membranes can be based on graphene or transition metal dichalcogenides (TMD) for example $\mathrm{MoS}_{2}, \mathrm{NbS}_{2}, \mathrm{NbSe}_{2}, \mathrm{TaS}_{2}$ etc and other $2 \mathrm{D}$ materials like $\mathrm{BN}$. The graphene membrane may be made by a variety of methods like liquid phase method, vapor phase method, polymer 
method and other methods that can grow the grain boundaries as shown in Figure 1(a). The pore size and spacing in the grain boundaries and the channels can be different in the layers to increase the separation selectivity of particular substances as seen in Figure 1(b).

(a)

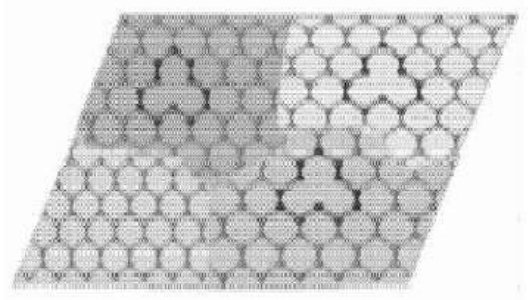

(b)

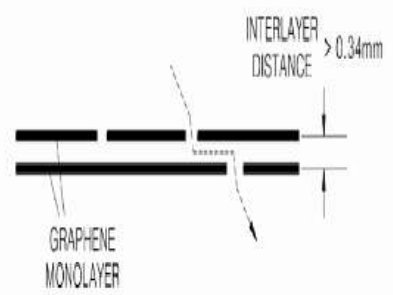

Fig.1. Schematic illustration of different forms of grain boundaries and pores in (a) graphene layer (b) cross-section of a bilayer (Choi et al 2012)

The pores can have a width of about $0.335 \mathrm{~nm}$ to $100 \mathrm{~nm}$. The pore size and channels can be controlled by adjustments to the graphene growth rate or by changing the carbon supply source to methane or hydrocarbon-based organic polymers. These pores can be made so small as to selectively pass only water molecules and block the hydrated salt ions in desalination. In addition to pores arising from grain boundaries, fine pores could be formed on the graphene membrane as a result of defects near 5- or 7-membered rings as depicted in Figure 2.

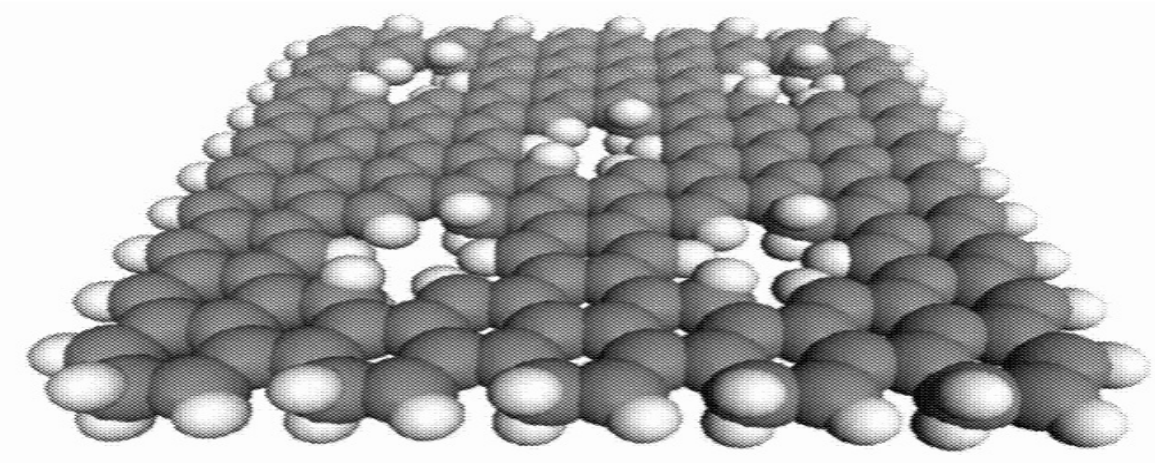

Fig.2. Schematic illustration of structure of graphene monolayer including defects (Choi et al 2012)

The procedure and description above of forming nanopores (Choi et al 2012) is not exclusive to graphene but also applies to the other 2D materials for example $\mathrm{MoS}_{2}$ and $\mathrm{BN}$.

It is difficult to remove and transfer atomically thin layers of $2 \mathrm{D}$ materials containing nanopores from the growth substrate as it can give rise to tear and conformity problems. This issue is addressed by Sinsabaugh et al 2015. The process involves manipulation of the $2 \mathrm{D}$ material such as graphene by first providing a support layer while it is still adhered to the growth substrate, then, second release of the substrate from the $2 \mathrm{D}$ material resulting in a $2 \mathrm{D}$ material attached to the support layer. Figure 3 
illustrates this 3 step process; the operations of forming, depositing a support layer and removal of the substrate.
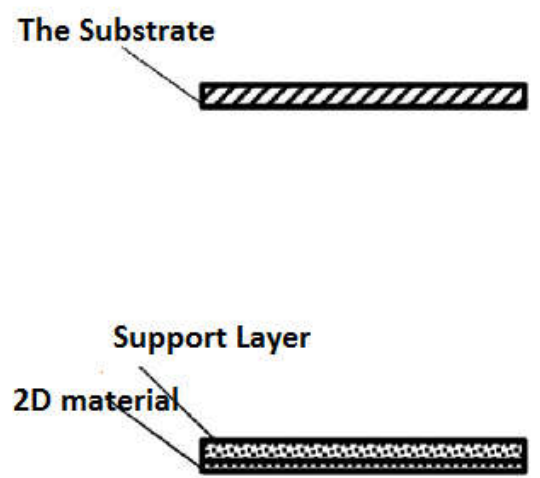
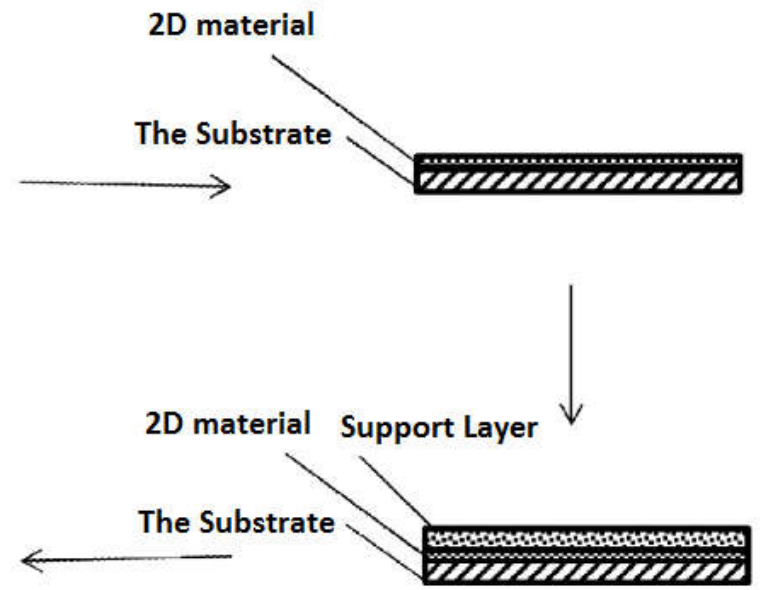

Fig.3. Schematic illustration of the process of formation on substrate, deposition of support layer and removal of substrate (Sinsabaugh et al 2015)

The substrate can be metal like $\mathrm{Cu}$ or $\mathrm{Ni}$ and the removal of the substrate is achieved by etching with a suitable etchant, like ammonium persulphate. The method uses a non-sacrificial porous support layer that can contain a plurality of pores with a pore size gradient in some embodiments. Such an embodiment has a smaller pore size in the supporting layer close to the $2 \mathrm{D}$ material surface compared to the opposite end. This feature can be achieved by electro-spinning of fibres with a density that is higher near the 2D material surface and lower further away from the 2D surface. Various chemical and physical techniques for perforating graphene or the 2D material can be employed like particle bombardment, chemical oxidation, lithographic patterning or a combination of these (Lehtinen et al 2011; Hern et al, 2014; Gilbert et al 2017). This can be done while it is adhered to the growth substrate or after removal of growth substrate and while it is attached to the supporting layer.

The most recent invention is of Stoltenburg et al 2017. The method consists of making a composite film of an atomically thin material (such as graphene) and a polymer layer; bombarding the same to create a plurality of pores in at least the graphene layer. The atomically thin material could be any of the following: a few layers of graphene, molybdenum disulfide, boron nitride, hexagonal boron nitride, niobium diselenide, silicene, or germanene. The best mode for the process of making and etching the nanoporous membrane and the execution of the invention is illustrated in Figure 4. The composite film consists of an atomically thin layer of 2D material and polymer film. A hot press manufacturing process could be used to make the composite film. The polymer film is polycarbonate with thickness ranging from 25-250 microns. Materials like polyester, polypropylene, polyimide, polymethyl methacrylate or polyvinylidene fluoride could be used for the polymer film. 


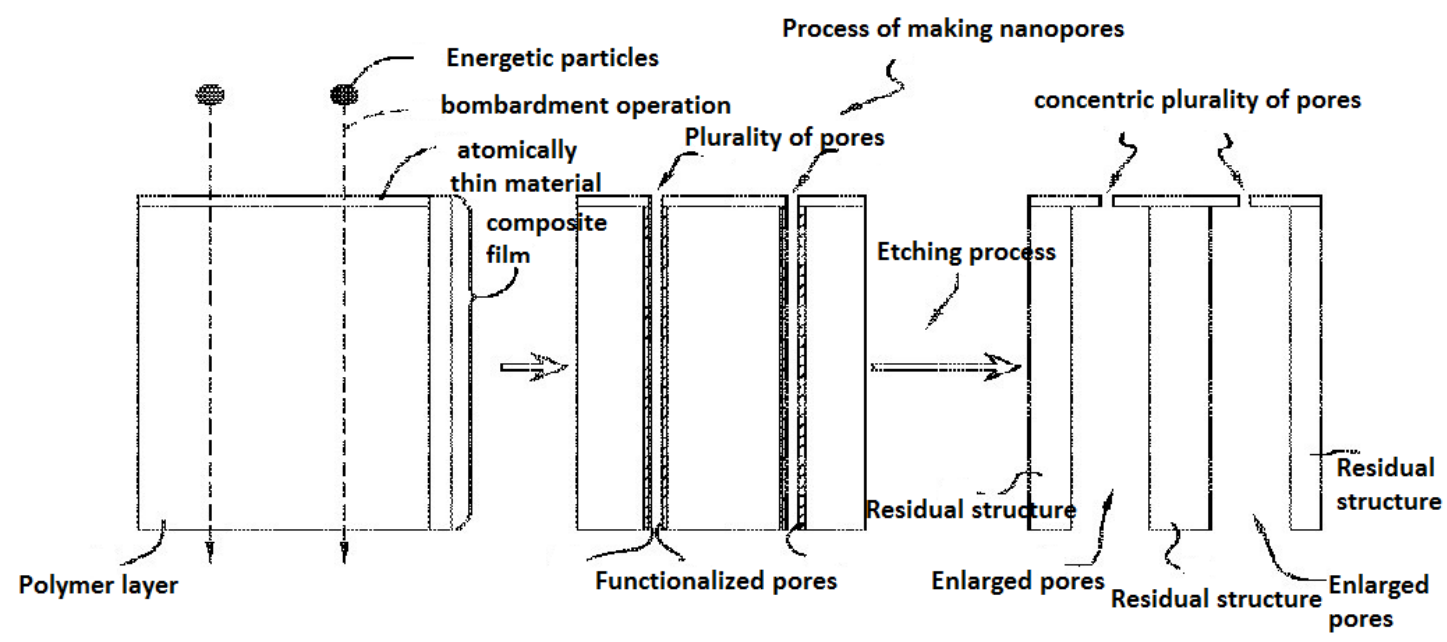

Fig.4. A schematic diagram of a process for making a nanoporous membrane with an initially non-porous polymer film (Stoltenburg et al 2017)

The energetic particles may be electrons, neutrons, ions, ion clusters or similar particles that are sufficiently energetic to traverse the composite film. Typical energies $>1 \mathrm{MeV} /$ micron thickness are directed at the composite film during the bombardment operation. Another aspect is the selection of the energetic particles to form a plurality of pores in the composite film such that the chemical nature of polymer layer is changed and the functionalizing of the pore occurs. Upon completion of the bombardment process the composite film undergoes the etching process. The entire film is immersed in an appropriate etching fluid, which depends on the type of polymer film used. For polycarbonate a solution of $\mathrm{NaOH}$ is used for a predetermined length of time. During the etching process the etchant attacks the chemical functionalizing of the polymer film in the pores to remove the chemical functionalized area and form enlarged pores in the polymer film. Depending upon the extent of chemical functionalization and the etching parameters the pore size can be controlled and it may range from 10-1000 nm. A residual polymer structure is formed as a result of the etching process. The end results of the etching process provides a plurality of pores that have a size ranging from 0.5 to $10 \mathrm{~nm}$ in graphene that are concentrically aligned with the enlarged pores. Thus the nanoporous membrane (active layer) has a one-to-one mapping of the holes with the polymer layer (substrate). An alternate method of producing a nanoporous membrane is depicted in Figure 5. The method is similar to above described method except that the polymer film is already porous with enlarged pores. The composite film (which consists of the atomically thin layer and polymer layer) is the same as the composite film in the previous method and has the same characteristics. The energetic particles during the bombarding process are selected such that there is no chemical functionalization of the polymer film and it is inert to the pore enlargement. The process of bombardment creates a plurality of pores in graphene or other atomically thin material that may or may not be concentric with the enlarged polymer pores. Further, it may form pores that extend partially into the polymer layer to create a cavity. The process may also create pores that extend all the way through the graphene and the polymer layer i.e. the composite layer. 


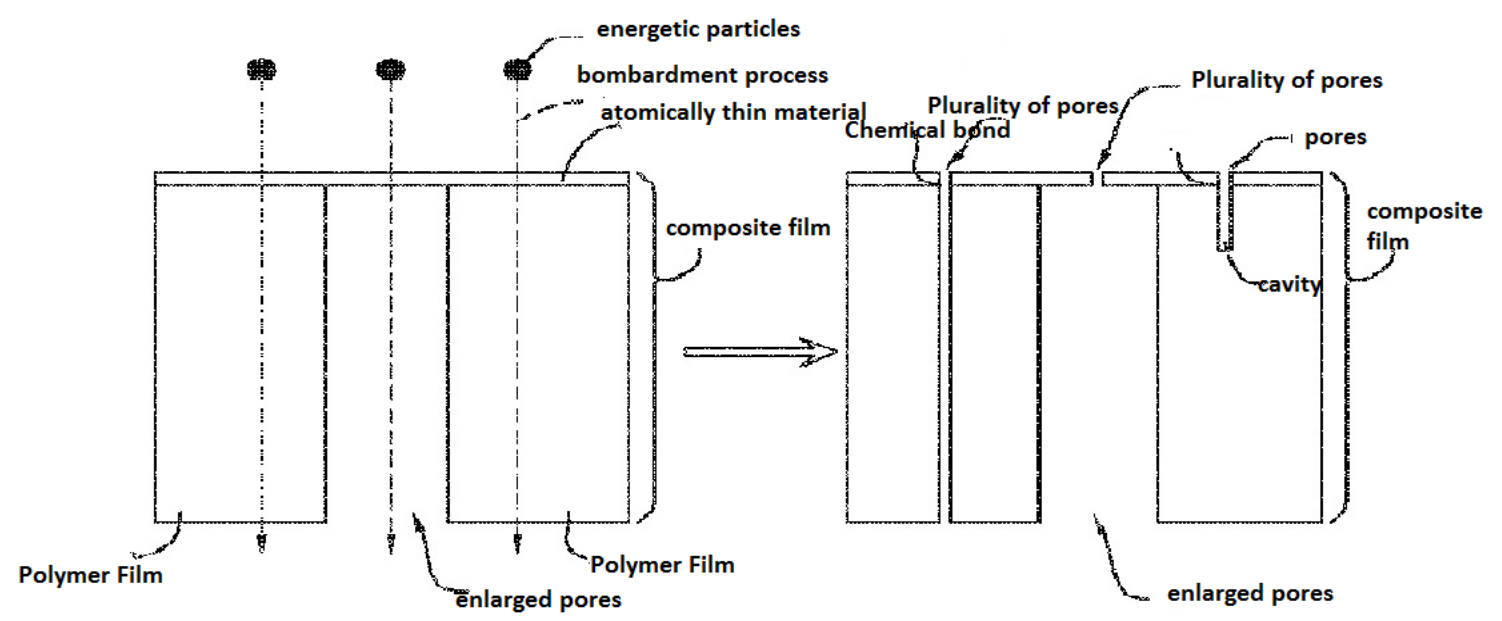

Fig.5. Schematic diagram of a process for making a nanoporous membrane with an initially porous polymer film

(Stoltenburg et al 2017)

Furthermore, a chemical bond may be formed between the graphene and polymer layer that will secure it and strengthen the composite film and support the graphene membrane. As a result, there can be alignment of the pores of size $0.5-10 \mathrm{~nm}$ with the enlarged pores to provide higher permeability. This method has the advantage that the particles for bombardment can be different from those needed to create tracks in the polymer film, thereby giving more flexibility in forming the composite film. Whereas the advantage of the previous method (Fig. 4) allows for simultaneous creation of the holes in both graphene and the polymer film that are one to one and are concentric thereby considerably increasing permeability of the composite film and manufacturability. Processing active layer (graphene) and support layer (polymer film) simultaneously is easier and more scalable. Having discussed the various types of nanopores and the patents involved in the making these nanoporous membranes the application to desalination will be discussed in the next section.

\section{Process, performance and effectiveness of nanopores and patents}

Several membrane and pressure driven technologies like RO, NF, Ultrafiltration (UF) and Microfiltration (MF) are in use at present (Pendergast and Hoek 2011; Khin et al 2012; Goh et al 2013). 19\% of the world has RO installations (IDA 2008) and it is the conventional method in use. RO requires high pressures and the energy consumption costs equal half the total cost; the desalination of $1 \mathrm{~m}^{3}$ of seawater requires $3.4 \mathrm{kWh}$ of energy at $6.5 \mathrm{MPa}$ (Fritzmann et al 2007; Semait 2008). Seawater predominantly contains $\mathrm{Na}^{+}$(Sodium) and $\mathrm{Cl}^{-}$(Chlorine) ions and has an average salinity of $3.5 \%(35 \mathrm{~g} / \mathrm{L})$ of dissolved salts. Typical values of the various ions present in the Arabian Gulf seawater in ppm (parts per million) is shown in Table1. Moderate amounts of $\mathrm{SO}_{4}{ }^{2-}$, $\mathrm{Mg}^{2+}, \mathrm{K}^{+}$and $\mathrm{Ca}^{2+}$ ions are also present. The table also shows the radii of the hydrated ions for the main components from references (Baldanov et al 2006; Tanganov 2013). The mesh size has an inverse relation to the cost so an estimation of the size of the hydrated ions in sea water is very useful. 
Table 1 Arabian Gulf Seawater composition adapted from (Baldanov et al 2006; Tanganov 2013).

\begin{tabular}{|c|c|c|c|}
\hline Ion & Conc. $[\mathbf{p p m}]$ & Ion radius [nm] & Hydrated ion radius [nm] \\
\hline $\mathrm{Na}^{+}$ & 14,161 & 0.098 & 0.360 \\
\hline $\mathrm{Cl}^{-}$ & 25,491 & 0.181 & 0.270 \\
\hline $\mathrm{SO}^{2-}$ & 3,594 & 0.147 & 0.300 \\
\hline $\mathrm{Mg}^{2+}$ & 1,642 & 0.078 & 0.395 \\
\hline $\mathrm{K}^{+}$ & 722 & 0.133 & 0.315 \\
\hline $\mathrm{Ca}^{2+}$ & 530 & 0.106 & 0.348 \\
\hline
\end{tabular}

As seen from the table the radii of hydrated ions are almost twice the radius of the water molecule which is $0.138 \mathrm{~nm}$. The knowledge of the values of the hydrated ions helps in the manufacture of membranes with optimal size and the costs of devices for desalination is reduced as a result of the lower pressures involved. The choice of the average pore size depends on the desired result of excluding a particular species and in turn is dependent on the size of the particular hydrated ion. Selection of a membrane material is based on the fact that it should be thin to maximize flow rate, chemically inert and stable and mechanically strong, with size-defined pore that provides selectivity by blocking large molecules and allowing smaller ones. The bright future for desalination with novel nanoporous membranes like graphene and the related materials and the tremendous progress and promise shown in this direction is discussed with the related patents (Stetson et al 2013; Grossman et al 2014; Mahurin et al 2016 and Li and Ruhong 2015).

In the process described by Stetson et al (2013), a perforated graphene membrane separates sodium, chlorine and other ions from water. The apertures in the membrane are designed to pass water molecules and to not pass the smallest $\mathrm{Na}^{+}, \mathrm{Cl}^{-}$and other relevant ions. The deionised water flowing through the graphene membrane is collected. Figure 6 shows a notational representation of this disclosure using a perforated graphene sheet.

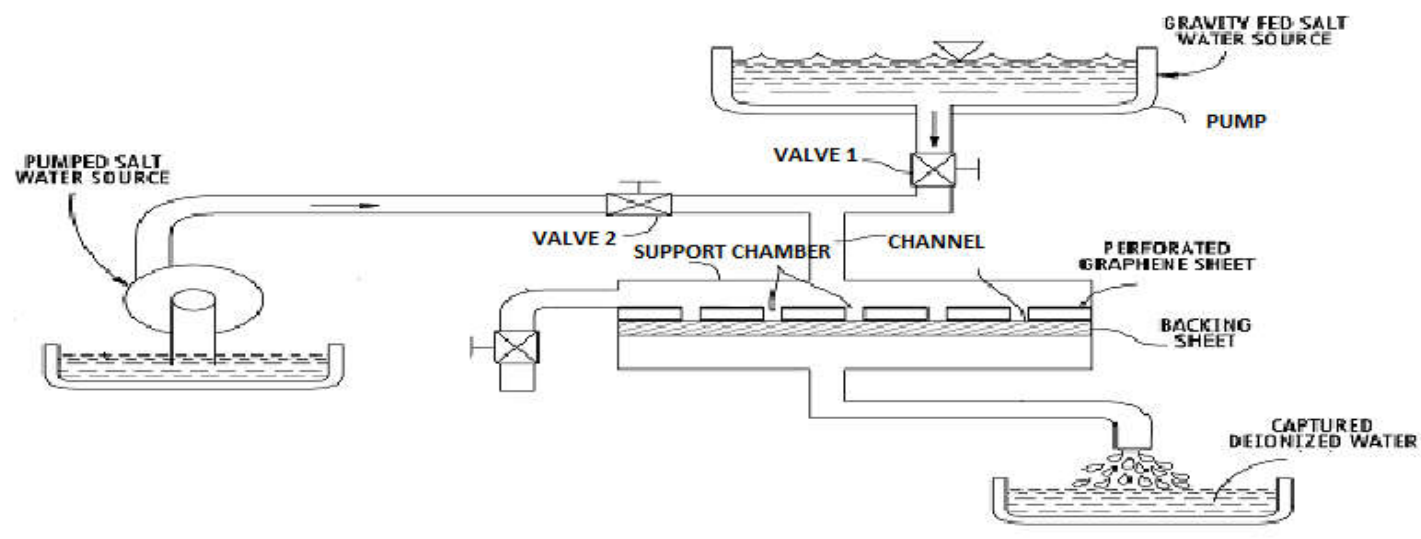

Fig.6. A notational representation of a water filter, using a perforated graphene sheet (Stetson et al 2013) 
A channel conveys the ion-laden water to a filter membrane mounted on a supporting chamber. The ion-laden water may be seawater or brackish water. The filter membrane can be wound into a spiral in a known manner. Flow impetus or pressure of the ion-laden water flowing through channel of Figure 6 can be provided either by gravity from a tank or from a pump. Valves 1 and 2 allow for the selection of the source of ion-laden water. In the apparatus or arrangement, the filter membrane is a perforated graphene sheet. A planer view of the same is shown in Figure 7 below.

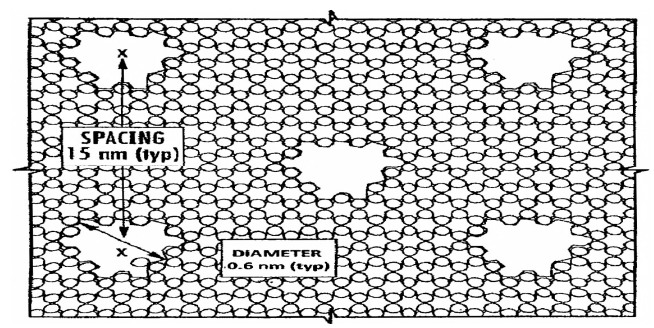

Fig.7. A plan view of a perforated graphene sheet, showing $0.6 \mathrm{~nm}$ diameter perforations or apertures and interperforation dimensions (Stetson et al 2013)

The ionized water flows first through a graphene layer dimensioned with pores allowing chlorine ions to pass and then through a second graphene layer with pores designed to pass $\mathrm{Na}^{+}$ions. The concentrated $\mathrm{Cl}^{-}$and $\mathrm{Na}^{+}$ions accumulating on the graphene layers can be separately harvested.

Mahurin et al (2016) also used nanoporous graphene for desalination. They have devised a process for the flow of salt water through a free standing graphene layer, having pores up to a size of $1 \mathrm{~nm}$ and with pore edges passivated by silicon. When water flows through the first planer side, the salt ions are blocked and salt free desalinated water exits from the other side. The membrane is supported on a substrate that contains a window and the free standing graphene layer spans this window and the salt water flows only through this portion. Figure 8 shows the single graphene layer suspended over the hole /window in the support material.

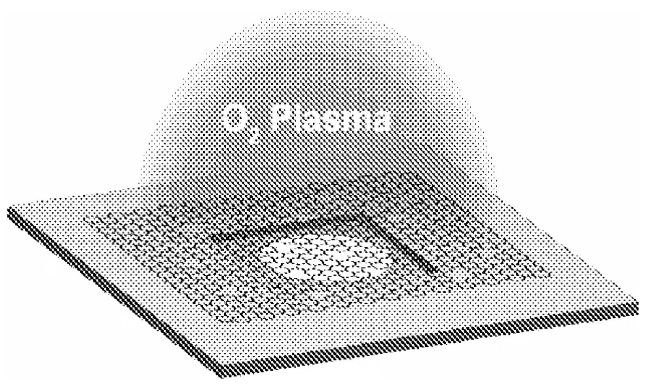

(a)

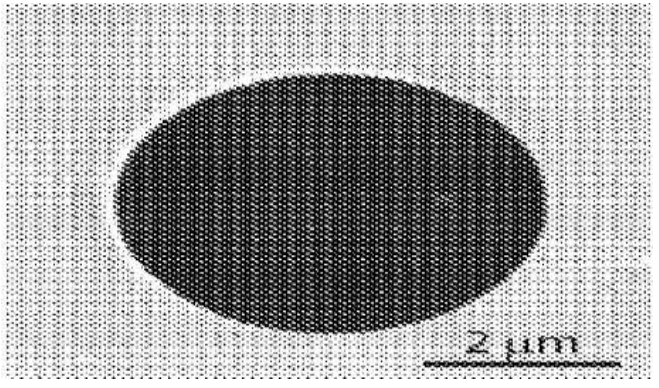

(b)

Fig.8. (a) Schematic of single layer graphene being subjected to oxygen plasma etch process while suspended over a hole in a support material (b) SEM image of single-layer Graphene suspended over a hole in a support material (Mahurin et al 2016)

Seawater contains at least one of sodium or potassium ions and at least $10 \mathrm{~g} / \mathrm{L}$ of the salt species, and the removal of salts is $\sim 95-99 \%$. The membrane is disposed on the opening of the container, which 
is inverted to feed seawater by gravity to the first planer side, resulting in desalinated water flowing out of the second planer side. The seawater is fed continuously into the container.

Grossman et al (2014) used a variety of porous materials, including nanoporous materials for filtration, purification and/or separation applications. The materials may be thin, flexible, and manufactured with a control over pore size and spatial distribution for applications in desalination. These could be a nano-structured carbon material, such as carbon nanotubes, graphite, graphene, or graphene oxide. For example the porous material could be a single layer or multiple layers of graphene arranged on a support or formed on a substrate that can be fabricated to include a plurality of pores having an average pore size of about $1 \mathrm{~nm}$ or less. The pores can be made to have an optimal size so as to effectively reject certain ions species, such as $\mathrm{Na}^{+}$and $\mathrm{Cl}^{-}$and allow the flow of other species e.g. water molecules. Nanoporous graphene materials having an overall porosity of $10 \%$ and containing a plurality of pores with an average pore size of about $6 \AA$ may exhibit a water permeability of about $50 \mathrm{~L} / \mathrm{cm}^{2} /$ day/MPa to about $60 \mathrm{~L} / \mathrm{cm}^{2} /$ day/MPa, which is two to three orders of magnitude higher than known UF, NF and reverse osmosis membranes. Functionalization in addition to pore size, affects the permeability of the water and the salt rejection performance of the membrane. As shown by (Sint et al 2008), the chemical functionalization of graphene nanopores can tune and selectively reject certain solvated ions. The carbon atoms at the edge of a pore on a graphene membrane can be functionalized by hydrophilic groups-OH or hydrophobic groupshydrogen, alkyl, aryl etc or by substituted/un-substituted amino etc. The hydrophilic functionalization allows faster water flow by providing a smoother entropic landscape for the water molecules whereas, hydrophobic functionality at or near the pore edges with hydrogen may enhance salt rejection. The key performance indicators of any desalination technology are salt rejection and water flux and Fig.9 illustrates the high performance of graphene nanopores as compared to RO other common desalination methods.

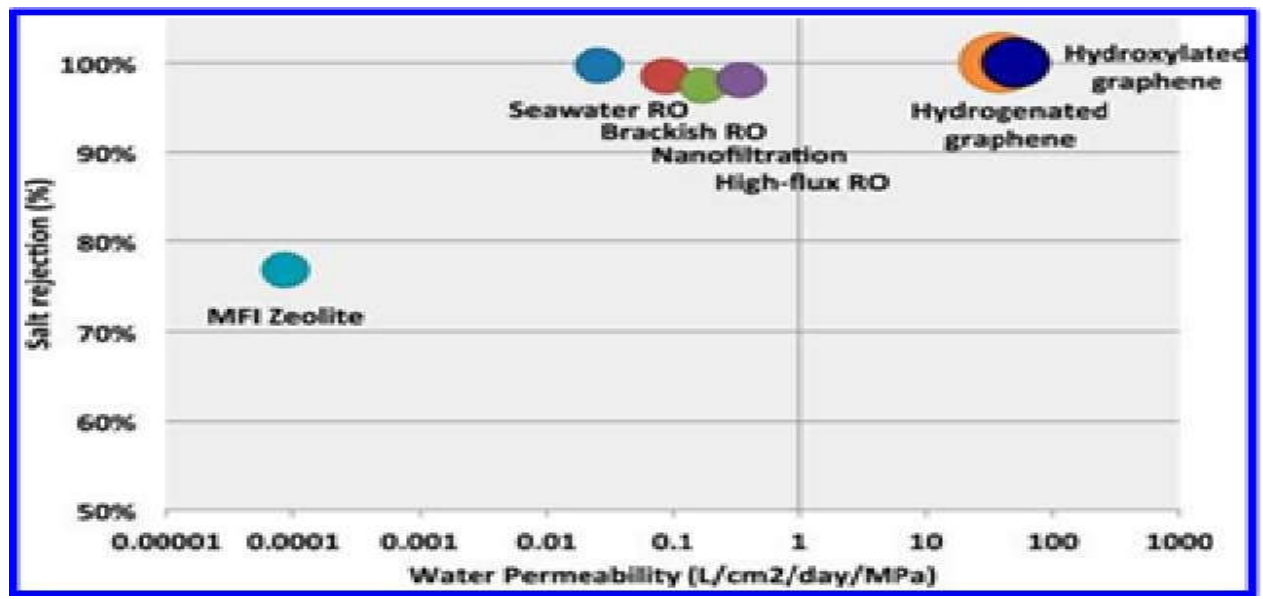

Fig.9. The two most important key performance parameters, salt rejection on y axis and water flux/permeability on $\mathrm{x}$ axis of graphene nanopores compared with the other common desalination technologies; reproduced with permission from Cohen-Tanugi, and Grossman copyright (2012) ACS.

In another patent, $\mathrm{Li}$ and Ruhong (2015) use mechanical strain control to open and close pores and tune a $2 \mathrm{D} \mathrm{MoS}$ desalination membrane. This invention offers more flexibility compared to graphene which has a fixed open/closed state that cannot be tuned externally. The sandwich like structure of a 
ML of $\mathrm{MoS}_{2}$ has a Young's modulus of $\sim 270 \mathrm{GPa}$ which is significantly smaller than the graphene sheet-like structure of a single layer of about $1 \mathrm{TPa}$. It is therefore more sensitive to mechanical strain and $\mathrm{MoS}_{2}$ nanopores and can be made controllable with "open" and "closed" states, i.e. the pore opening size can be changed to allow or stop water flow by using strain. The steps involved in strain controlled $\mathrm{MoS}_{2}$ as desalination membrane is first- to fix the $\mathrm{MoS}_{2}$ nanoporous membrane on a frame, second- install the frame-film device filter in seawater, third-apply a pull force to the frame to increase the membrane frame surface area about 6-12\% and fourth to pass pressurised seawater with pressure range $0-100 \mathrm{MPa}$ through the $\mathrm{MoS}_{2}$ membrane to allow water molecules to pass and block the salt ions and thus complete the desalination process. In some embodiments, the $\mathrm{MoS}_{2}$ monolayer can have Mo or S or both Mo and S vacancy defects for selectivity and improving the performance.

\section{Challenges and outlook}

Nanopores, with the low energy requirements and high desalination efficiency are proving to be the new generation desalination devices. The theoretical studies of graphene and low dimensional TMDs give insight to numerous possibilities and predict the device characteristics for efficient desalination. The low pressure operation of these nanoporous membranes and the self cleaning, no fouling characteristics make them very attractive for use in desalination. Graphene, BN, $\mathrm{MoS}_{2}$ and related nanopores offer a safe, reliable, sensitive, energy saving and cost-effective water desalination technology that is ready to be put into action. But, in order to translate this technology to large scale production various aspects concerning process and fabrication need to be addressed. The main challenges are large scale defect free well defined membrane synthesis, uniform, precise and small size pore generation, mechanical stability and functionalization of membranes. Liu et al (2014) successfully generated nanopores of 1-10 nm diameters in $\mathrm{MoS}_{2}$ using a transmission electron microscope with a highly focused electron beam. Large-area, good quality membrane with nanopores in the nm and sub-nm can be fabricated as shown by Waduge et al (2015) and Feng et al (2015). Graphene is the ideal membrane with its only one atom layer thickness and the simple techniques for introducing nanopores by ionic irradiation or chemical treatment can be implemented with great success. The simulation study of Jun et al (2011) found that graphene mono-layers pinned every 40-160 A can withstand pressures greater than $500 \mathrm{MPa}$ without ripping. Experimental results for bulge tests ((Bunch et al 2008) also confirmed the high strength of graphene. The rapid experimental progress suggests the practical feasibility of accurate and large scale synthesis of highly ordered nanoporous graphene and related 2D materials and the commercialization of this desalination technology is in the immediate future.

\section{Acknowledgements}

This work was not supported by any funding. The authors would like to take the opportunity to thank Eng. Wajeeh M. A. Aqra for his continuous encouragement and support.

\section{References}


Baldanov M., Baldanova D., Zhigzhitova S., \&Tanganov B. 2006 To the problem of the radii of hydrated ions, Reports of the Russian High School Academy of Science 32-34

Choi, J-Y. \& Park B-H. 2012 Separation membrane including graphene. U.S. Patent, 0255899. https://patents.google.com/patent/US20120255899 (accessed 25 May 2019)

Dumcenco D. Ovchinnikov D., Marinov K., Lazić P., Gibertini M., Marzari N., Lopez Sanchez O, Kung Y.C., Krasnozhon D., Chen M.W., Bertolazzi S., Gillet P., Fontcuberta i Morral A., Radenovic A. \& Kis A. 2015 Large-area epitaxial monolayer MoS2. ACS Nano 9(4), 4611-4620. Elimelech M. \& Phillip W. A. 2011 The Future of Seawater Desalination: Energy, Technology, and the Environment. Science, 333, 712-717.

Feng, J., Liu K., Graf M., Lihter M., Bulushev R.D., Dumcenco D., Alexander D.T.L., Krasnozhon D., Vuletic T., Kis A. \& Radenovic A. 2015 Electrochemical reaction in single layer $\mathrm{MoS}_{2}$ : nanopores opened atom by atom. Nano Lett. 15(5), 3431-3438.

doi:10.1021/acs.nanolett.5b00768

Fritzmann C., Löwenberg J., Wintgens T., \& Melin T. 2007 State-of-the-art of reverse osmosis desalination. Desalination, 216(1), 1-76. doi:10.1016/j.desal.2006.12.009

Gilbert S. M. , Dunn G., Pham T., Shevitski B., Dimitrov E., Aloni S. \& Zettl A. 2017

Fabrication of Atomically Precise Nanopores in Hexagonal Boron Nitride. DOI: 10.1038/s41598017-12684-x

Goh P. S., Ismail A. F., Ng B. C. 2013 Carbon Nanotubes for Desalination: Performance Evaluation and Current Hurdles. Desalination, 308, 2-14. doi:10.1016/j.desal.2012.07.040 Grossman J., Ferralis N., David Tanugi D-C., \& Dave S.H. 2014 Porous materials and methods including nanoporous materials for water filtration. Google patents, WO 2014152407. https:/patents.google.com/patent/WO2014152407A1/en?oq=WO+2014152407 (accessed 25 May 2019)

Heiranian M., Farimani A.B. \& Aluru N.R. 2015 Water desalination with a single-layer http://www.freepatentsonline.com/y2016/0207798.html (accessed 25 May 2019) https://patents.google.com/patent/CN104925907A/en?oq $=\mathrm{CN}+104925907 \mathrm{~A}+$ (accessed 25 May 2019)

International Desalination Association (IDA), Desalination in 2008 global marker snapshot, 21 st GWI/International Desalination Association Worldwide Desalting Plant Inventory2008.

http://idadesal.org/wp-content/uploads/2008/10/2008ida-desalination-snapshot_october-2008.pdf (accessed 25 May 2019)

Jeon, J. Sung Kyu Jang S.K., Su Min Jeon S.M., Gwangwe Yoo G., Yun Hee Jang Y.H., JinHong Park J-H. \& Sungjoo Lee S. 2015 Layer-controlled CVD growth of large-area twodimensional $\mathrm{MoS}_{2}$ films. Nanoscale 7, 1688-1695.

Khin M.M., Nair A.S., Babu V. J., Murugan R., \& Ramakrishna S. 2012 A review on nanomaterials for environmental remediation. Energy Environ. Sci. 5 (8) 8075-8109. Doi: $10.1039 / \mathrm{c} 2 \mathrm{ee} 21818 \mathrm{f}$

Li W. \& Ruhong W. 2015 Application of nano-porous molybdenum disulfide controlled by mechanical strain to sea water desalination. Google patents, CN 104925907A.

Li W., Yang Y., Weber J.K., Zhang G., \& Zhou R. 2016 Strain-Controlled Nanoporous $\mathrm{MoS}_{2}$ Filter for Water Desalination. ACS Nano 10, 1829-1835. doi:10.1021/acsnano. 
Liu K., Feng J. D., Kis A. \& Radenovic A. 2014 Atomically thin molybdenum disulfide nanopores with high sensitivity for DNA translocation. ACS Nano, 8, 2504-2511.

Mahurin S.M., Vlassiouk I., Dai S., Surwade S.P., Unocic R.R., \& Smirnov S. 2016 Nanoporous graphene membrane for desalination of salt water. US Patent, 0207798.

http://www.freepatentsonline.com/y2016/0207798.html (accessed 25 May 2019)

Moghadam F. \& Park H.B. 2018 Two-dimensionalmaterials:an emerging platform for gas separation membranes. Current Opinion in Chemical Engineering, 20, 28-38.

MoS2nanopore. Nature Communications, 6 Article no. 8616. DOI: 10.1038/ncomms9616

Nguyen B.H. \& Nguyen V.H. 2016 Promising applications of graphene and graphene based nanostructures. Adv. Nat. Sci: Nanosci. Nanotechnol. 7, 023002

Pendergast M.M. \& Hoek E.M.V. 2011 A review of water treatment membrane

nanotechnologies. Energy and Environmental Science, 4 (6), 1946-1971.

Ramanathan A.A., Aqra M.W. \& Al-Rawajfeh A.E. 2018 Recent advances in 2D-nanopores for desalination. Environ Chem Lett. DOI: 10.1007/s10311-018-0745-4

Semiat R., 2008 Energy issues in desalination processes, Environ. Sci. Technol. 42(22) 81938201. doi:10.1021/es801330u

Sinsabaugh S.L., Bedworth P.V., Casey Jr. D.F., Heise S.E., Sinton S.W. Stoltenberg R.M., \& Swett J.L. 2015 Process for forming composite structures with a two-dimensional material using a porous, non-sacrificial supporting layer.

http://www.freepatentsonline.com/y2015/0217219.html (accessed 25 May 2019)

Sint K., Wang B., \& Král P. 2008 Selective ion passage through functionalized graphene nanopores. J. Am. Chem. Soc. 130(49), 16448-16449. doi:10.1021/ja804409f

Stetson J.B., Mercurio J., Alan Rosenwinkel A., Bedworth P.V. 2013 Perforated graphene deionization or desalination. US patent, $8361321 \mathrm{~B} 2$.

https:/patents.google.com/patent/US8361321?oq=US+8361321+B2 (accessed 25 May 2019)

Stoltenburg R.M., Bedworth P.V., Heise S.E. \& Sinton S.W. 2017 Nanoporous membranes and methods for making the same. U.S. Patent, 20170043300.

https:/patents.google.com/patent/US20170043300 (accessed 25 May 2019)

Suk M. E., \& Aluru N.R. 2010 Water Transport through Ultrathin Graphene. J. Phys. Chem. Lett. 1, 1590. doi:10.1021/jz100240r

Suk M. E., Raghunathan A.V., \& Aluru N. R. 2008 Fast reverse osmosis using boron nitride and carbon nanotubes. Appl. Phys. Lett., 92, 133120. doi:10.1063/1.2907333.

Surwade S.P., Smirnov S.N., Vlassiouk I.V., Unocic R.R., Dai S.,Veith G.M. \& Mahurin S.M. 2015 Water desalination using nanoporous single-layer graphene. Nature Nanotechnology 10, 459-464. doi:10.1038/NNANO.2015.37

Tamsyn A. Hilder T.A., Gordan D. \& Chung S-H. 2009 Salt Rejection and Water Transport Through Boron Nitride Nanotubes. small 5(19), 2183-2190.

Tanganov B. B. 2013 Modern problems of science and education. Available at https://worldscience.ru/en/article/view?id=31409 (accessed 25 May 2019)

Tanugi D. C. \& Grossman J. C. 2012 Water Desalination across Nanoporous Graphene. Nano Lett. 12 3602. doi:10.1021/nl3012853 
Tanugi D. C. \& Grossman J. C. 2014 Water permeability of nanoporous graphene at realistic pressures for reverse osmosis desalination. The Journal of Chemical Physics, 141074704.

doi:10.1063/1.4892638

Tanugi D. C., Lin L. \& Grossman J. C. 2016 Multilayer Nanoporous Graphene Membranes for Water Desalination. Nano Lett. 16 (2) 1027-1033. doi:10.1021/acs.nanolett.5b04089

Thanh T.D., Chuong N.D., Hien H.V., Kshetri T., Tuan L.H., Kim N.H., \& Lee J.H. 2018 Recent advances in two-dimensional transition metaldichalcogenides-graphene heterostructured materials forelectrochemical applications. Progress in Materials Science 96, 51-85.

Waduge P., Bilgin I., Larkin J., Henley R.Y., Goodfellow K., Graham A.C., Bell D.C.,

Vamivakas N., Kar S. \& Wanunu M. 2015 Direct and scalable deposition of atomically thin lownoise $\mathrm{MoS}_{2}$ membranes on apertures. ACS Nano, 9(7), 7352-7359. doi:

10.1021/acsnano.5b02369

Wali F. 2014 The future of desalination research in the Middle East.

https://www.natureasia.com/en/nmiddleeast/article/10.1038/nmiddleeast.2014.273 (accessed 25

May 2019) doi:10.1038/nmiddleeast.2014.273

Wasfi A, Awwad F and Ayesh A.I. 2018 Graphene-based Nanopore Approaches for DNA Sequencing: A Literature Review. Biosensors \& Bioelectronics, 119, 191-203. DOI: 10.1016/j.bios.2018.07.072

Wu Y. B., Wagner L. K., \& Aluru N. R. 2015 The interaction between hexagonal boron nitride and water from first principles. J. Chem. Phys., 142, 234702. doi: 10.1063/1.4922491

Yuan H., Abu-Reesh I.M., \& He Z. 2016 Enhancing Desalination and Wastewater Treatment by Coupling Microbial Desalination Cells with Forward Osmosis. Chemical Engineering Journal, 270, 437-443. Doi:10.1016/j.cej.2015.02.059

Zhu C. Li H., Zeng X.C., Wang E.G. \& Meng S. 2013 Quantized Water Transport: Ideal Desalination through Graphyne-4 Membrane. Scientific Reports 3, Article number: 3163. Doi: 10.1038/srep031632013 